\title{
Hydration States and Blood Compatibility of Hydrogen-Bonded Supramolecular Poly(2-methoxyethyl acrylate)
}

Jankova, Katja; Javakhishvili, Irakli; Kobayashi, Shingo; Koguchi, Ryohei; Murakami, Daiki; Sonoda, Toshiki; Tanaka, Masaru

\section{Published in:}

ACS Applied Bio Materials

Link to article, DOI:

10.1021/acsabm.9b00363

Publication date:

2019

Document Version

Peer reviewed version

Link back to DTU Orbit

Citation (APA):

Jankova, K., Javakhishvili, I., Kobayashi, S., Koguchi, R., Murakami, D., Sonoda, T., \& Tanaka, M. (2019). Hydration States and Blood Compatibility of Hydrogen-Bonded Supramolecular Poly(2-methoxyethyl acrylate). ACS Applied Bio Materials, 2(10), 4154-4161. https://doi.org/10.1021/acsabm.9b00363

\section{General rights}

Copyright and moral rights for the publications made accessible in the public portal are retained by the authors and/or other copyright owners and it is a condition of accessing publications that users recognise and abide by the legal requirements associated with these rights.

- Users may download and print one copy of any publication from the public portal for the purpose of private study or research.

- You may not further distribute the material or use it for any profit-making activity or commercial gain

- You may freely distribute the URL identifying the publication in the public portal 


\title{
Hydration states and blood compatibility of Hydrogen-bonded supramolecular poly(2- methoxyethyl acrylate)
}

\author{
Katja Jankova, ${ }^{1,2}$ Irakli Javakhishvili, ${ }^{3}$ Shingo Kobayashi, ${ }^{1}$ Ryohei Koguchi, ${ }^{1,4}$ Daiki \\ Murakami, ${ }^{1}$ Toshiki Sonoda, ${ }^{1}$ and Masaru Tanaka ${ }^{1 *}$ \\ ${ }^{1}$ Soft Materials Chemistry, Institute for Materials Chemistry and Engineering, Kyushu
}

University, Build. CE41, 744 Motooka Nishi-ku, Fukuoka, 819-0395, Japan

${ }^{2}$ Department of Energy Conversion and Storage, Technical University of Denmark,

Elektrovej, Build. 375, 2800 Kongens Lyngby, Denmark

${ }^{3}$ Danish Polymer Centre, Department of Chemical and Biochemical Engineering,

Technical University of Denmark, Build. 229, 2800 Kongens Lyngby, Denmark 


\author{
${ }^{4}$ AGC Inc. New Product R\&D Center, 1150 Hazawa-cho, Kanagawa-ku, Yokohama, \\ Kanagawa 221-8755, Japan \\ KEYWORDS: blood compatible polymer, hydrogen bonding, poly(2-methoxyethyl \\ acrylate), free-standing film, intermediate water, 2-\{3-(6-methyl-4-oxo-1,4- \\ dihydropyrimidin-2-yl)ureido ethyl methacrylate.
}

\begin{abstract}
The practical use of the viscous liquid polymer, poly(2-methoxyethyl acrylate) (PMEA) was expanded from thin films with excellent blood compatibility to thick coatings and free-standing films without essentially sacrificing its blood compatibility. This was undertaken by creating multiple hydrogen-bonding polymer
\end{abstract} networks by introducing a functional methacrylic monomer bearing a 6-methyl-2-ureido$4[1 \mathrm{H}]$-pyrimidone group in the PMEA backbone via free radical copolymerization. The hydrogen-bonded PMEA (H-PMEA) contained about 6 mol\% of the functional monomer in the copolymer. These functional monomers as physical cross-links are distributed in the PMEA matrix with a $T_{\mathrm{g}}$ of $-35^{\circ} \mathrm{C}$, making $\mathrm{H}-\mathrm{PMEA}$ solid rubber-like material with 
recoverable tensile strain. Additionally, mechanical tests revealed its tensile strength, and thermogravimetric analyses confirmed its higher thermostability. The dry and hydration states of H-PMEA were assessed by differential scanning calorimetry, contact angle, and atomic force microscopy measurements. Comparison with viscous PMEA was made. For the first time, we included PVC alongside with PET, the surface we usually use as a negative control, in the platelet adhesion test with human blood, and found out that 1.5 times more platelets adhered onto the PVC surface than onto the PET surface, while H-PMEA proved to have a clear edge. Thus, H-PMEA may serve as a suitable replacement of polymers in products contacting blood as it shows potential for making free-standing films, thick coatings, implants and articles with various geometries for the medicinal industry.

Introduction 
Compatibility with human blood is an important characteristic of materials used in medical devices. Poly(2-methoxyethyl acrylate) (PMEA) is an acrylic homopolymer having an ethylene oxide unit in the side chain. It has low toxicity and is FDA approved for medical use. This polymer boasts the largest share on coating market, is easy to synthesize and inexpensive, possesses excellent blood compatibility, low protein adsorption and denaturation. ${ }^{1-3}$ Intermediately hydrophilic, with the water contact angle of approximately 40 degrees, it is not soluble in water above the molecular weight (MW) of $4-5 \mathrm{kDa}$, but it is soluble in methanol as well as many other organic solvents. Being a viscous liquid has not obstructed PMEAs extensive use, especially in the Japanese industry, to coat various organic and inorganic surfaces to which the polymer adheres well. These properties made it very useful to coat with a thin film of liquid PMEA various stents, an artificial oxygenator, ${ }^{4}$ a bioartificial liver, heart, ${ }^{5}$ cell enrichment filter, etc., and thus to protect blood from contacting the substrate material. However, only thin films were possible to be produced from PMEA so far. When the thickness of the film is below 50 microns the films are not sticky. Usually, for coating purposes, PMEA with the number average MW of around 23,000 Da (18-25 kDa) has been used. ${ }^{1-3}$ The effect of 
the MW of PMEA on the interfacial structure and the blood compatibility was investigated very recently. ${ }^{6}$ PMEAs with higher MWs were found to have a very little (30 and $44 \mathrm{kDa}$ ) or even more (183 $\mathrm{kDa}$ ) reduced blood compatibility as compared to PMEA with $19 \mathrm{kDa}$. These PMEAs with higher MW were also viscous and sticky. Recently, charged PMEA microspheres were synthesized ${ }^{7}$ by aqueous precipitation polymerization with potassium peroxydisulfate, and were also turned into small freestanding films with thicknesses up to $1.2 \mathrm{~mm}$.

Liquid polymers can be transformed into higher viscosity products by using either chemical or physical cross-linking. The chemical crosslinks are introduced by curing where covalent bonds are formed. However, besides using an additional step of curing, the material cannot be processed afterwards. Non-covalent interactions are often the means of property improvement in polymer science. By introducing hydrogen bonding, metal complexation, ionic or hydrophobic interactions, etc. the supramolecular polymer, which can be recycled, is obtained. ${ }^{8-9}$ Hydrogen bonding is not the strongest among the non-covalent interactions, but has been employed to confer many new functionalities like stimuli-responsiveness ${ }^{10}$ and self-healing properties to polymeric materials. ${ }^{11-14}$ 
Acid-base interactions are often utilized in polymer electrolyte membranes for fuel cells to increase the strength and conductivity of the ionic polymers. ${ }^{15-17}$ We have previously exploited a strategy involving hydrophobic interactions both in aq. solutions ${ }^{18-19}$ and in bulk. ${ }^{20}$ Triblock copolymers with small amounts of outer blocks were designed, which could associate in bulk to build a morphology very similar to that of the thermoplastic elastomers, where hard segments act as physical cross-links distributed in a matrix of a low $T_{\mathrm{g}}$ polyether polymer - this way $\mathrm{Li}$ ion conducting solid polymer electrolytes comprising PEG or PEG/PEGPG soft segments together with polyethylene or polypentafluorostyrene hard segments could be produced. ${ }^{20-21}$

Introducing additional chemical or physical interactions in MEA would allow preparation of a solid PMEA, which in turn would make it possible to manufacture thick coatings and materials with various shapes without negatively affecting its blood compatibility. Since the mid-20 th century, plasticized PVC has been utilized to collect and store whole blood, and various blood bags, tubes, catheters, etc. have been produced. Usually, the sodium salt of citric acid is added to the blood 22 acting as an anticoagulant, and increasing the stability of blood with time. As the most harmful in 
PVC are plasticizers, new plasticizers other than phthalates (including citrates) are being employed. ${ }^{22-26}$ Polymers other than PVC are being developed as well. ${ }^{23-24}$ However, both new plasticizers and polymers are expensive. Additionally, some of the new plasticizers for PVC have a foul odour, cause allergic reactions and are leachable. ${ }^{25}$ Due to these drawbacks, alternative materials are constantly sought. RENOLIT Medical supplies polyolefins, ${ }^{23}$ while MELITEK provides XE film grades of PP with high purity and without plasticizers for medical and pharmaceutical applications. ${ }^{24}$ It is claimed that these non-PVC containing alternatives fulfil all the demands posed on blood compatible materials. However, these alternative solutions are not economical.

The goal of this work is to combine benefits of PVC and PMEA into a single platform in an attempt to mitigate the problem associated with the scarcity of materials suitable for blood-contacting applications. Thus, a strategy to convert the low viscosity and highly blood compatible PMEA into a solid polymer without substantially decreasing its blood compatibility by employing copolymerization of MEA with a strong hydrogenbonding monomer in a simple and facile manner is proposed. 


\section{Experimental}

\section{Materials}

The MEA monomer (Sigma-Aldrich) was passed through a pre-packed disposable column to remove an inhibitor. $\alpha, \alpha^{\prime}$-Azobis(isobutyronitrile) (AIBN) was recrystallized from methanol. All other solvents or chemicals were employed as received unless specified otherwise. PVC was kindly supplied from Sekisui co., Japan. AIBN and phosphate-buffered saline (PBS) were obtained from FUJIFILM Wako Pure Chemical Corporation, Japan. Bovine serum albumin (BSA) and human fibrinogen from Sigma-Aldrich, USA, and human whole blood for the platelet adhesion test from Tennessee blood services, USA were employed. The micro-BCA protein assay kit from Thermo Fisher Scientific, USA was used to evaluate the amount of adsorbed protein.

\section{Synthesis and molecular characteristics of polymers}

PMEA was prepared by free radical polymerization. The polymerization was conducted in 1,4dioxane at $75{ }^{\circ} \mathrm{C}$ for 6 hours making use of $\mathrm{AIBN}$ as the initiator. ${ }^{27-28}$ The polymer was precipitated from THF/hexane mixture, and dried in vacuo at $60{ }^{\circ} \mathrm{C}$ for several days. The functional 2-\{3-(6-methyl-4-oxo-1,4-dihydropyrimidin-2-yl)ureido\} ethyl methacrylate and its Hbonding copolymer with MEA (H-PMEA) were synthesized and analyzed as reported in the literature. ${ }^{29} \mathrm{H}$-PMEA copolymer prepared in this work contains $6 \mathrm{~mol} \%$ of the functional monomer according to ${ }^{1} \mathrm{H}$ NMR spectroscopy (Figure S1). The molecular weight characteristics of both polymers are presented in Table 1 . 
Table 1. Glass transition temperatures, static water contact angles and the molecular weight characteristics of the synthesized PMEA, H-PMEA, and the used PET substrate

\begin{tabular}{|l|c|c|c|c|c|c|}
\hline Polymer & $\begin{array}{c}T_{\mathrm{g} \text { dry }} \\
{ }^{\circ} \mathrm{C}\end{array}$ & $\begin{array}{c}T_{\mathrm{g}} \\
\text { hydrated, } \\
{ }^{\circ} \mathrm{C}\end{array}$ & $\begin{array}{c}M_{\mathrm{n}}{ }^{\mathrm{b}} \\
\mathrm{Da}\end{array}$ & $M_{\mathrm{w}} / M_{\mathrm{n}}$ & $\begin{array}{c}\text { Sessile } \\
\text { drop }\end{array}$ & $\begin{array}{c}\text { Captive } \\
\text { bubble }\end{array}$ \\
\hline PMEA & -35.0 & -50.1 & 22,000 & 2.81 & $42 \pm 2$ & $30 \mathrm{~s}$ \\
\hline H-PMEA & -33.3 & -41.5 & 25,800 & 2.13 & $65 \pm 2$ & $142 \pm 1$ \\
\hline PET & 76 & - & - & - & $80 \pm 1$ & $115 \pm 3$ \\
\hline
\end{tabular}

a Measurements (degree, mean $\pm S D, n=5$ ) of the polymer films (annealed overnight at $\left.60{ }^{\circ} \mathrm{C}\right)$

b Determined by SEC with a PS calibration curve

\section{Analyses}

Analytical techniques

${ }^{1} \mathrm{H}$ NMR spectra were acquired on JEOL $500 \mathrm{MHz}$ JNM-ECX and Bruker Avance 300

$\mathrm{MHz}$ spectrometers in DMSO- $d_{6}$ or $\mathrm{CDCl}_{3}$ at room temperature.

Molecular weights and polydispersity indices were estimated by Size-Exclusion

Chromatography (SEC) equipped with a triple detector array (refractive index, right 
angle laser light scattering, and viscometer) SEC system of Malvern Viscotek TDAmax and Tosoh TSKgel columns connected in a series of guard column $\mathrm{H}_{H R}-\mathrm{H}, \mathrm{GMH}_{H R}-\mathrm{H}$

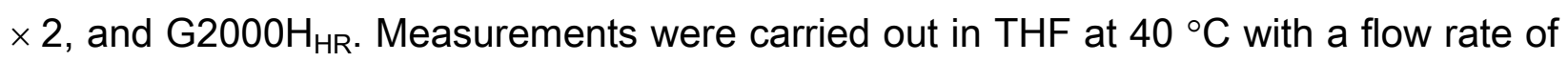
$1.0 \mathrm{~mL} / \mathrm{min}$. The system was calibrated using narrow molecular weight distribution PS standards in the range of $5 \times 10^{2}-3.64 \times 10^{5} \mathrm{~g} / \mathrm{mol}$.

Differential scanning calorimetry (DSC) measurement was conducted in the temperature range of $-100-50{ }^{\circ} \mathrm{C}$ and at the rate of $5.0{ }^{\circ} \mathrm{C} / \mathrm{min}$ on a Seiko Instruments Inc. X-DSC7000 purged with $\mathrm{N}_{2}$. $T_{\mathrm{g}}$ was determined from the second heating curve automatically, and is reported as the onset of the thermal transition. A TGA Q500 apparatus from TA Instruments was utilized to conduct thermogravimetric analyses (TGA). Samples were heated from 20 to $550{ }^{\circ} \mathrm{C}$ with a heating rate of $5{ }^{\circ} \mathrm{C} / \mathrm{min}$ under either air or nitrogen flow.

\section{Mechanical tests}

Films of H-PMEA and PVC with a thickness of $0.1-0.2 \mathrm{~mm}$ were hot pressed from each of the polymers between $2 \mathrm{PTFE}$ plates first at $60^{\circ} \mathrm{C}$ for 15 min under a pressure of $5 \mathrm{MPa}$, and at $150{ }^{\circ} \mathrm{C}$ for $15 \mathrm{~min}$ at $10 \mathrm{MPa}$, respectively, and then 5 more minutes at room temperature. 
Polymer films were drawn uniaxially at a rate of $10 \mathrm{~mm} / \mathrm{min}$ with a universal tensile testing machine (Tensilon RTC-1210A, A\&D Co., Ltd.) at room temperature. Dogbone specimens $64 \mathrm{~mm}$ long and $10 \mathrm{~mm}$ broad were used, and the measuring part was $10 \mathrm{~mm}$ long. Quantification of water-polymer interactions In hydrated macromolecules, 3 kinds of water are determined:27,28,30 non-freezing water $(N F W)$, intermediate water $(I W)$, and free water $(F W)$, and their content is given as NFWC, IWC, and FWC, respectively. DSC is employed to estimate the equilibrium water content (EWC) of a polymer as the water content at which a peak at about $0{ }^{\circ} \mathrm{C}$ corresponding to the melting of ice and a small shoulder or peak below this temperature originating from melting of $I W$ appear, and is given in equation (1):

$\operatorname{EWC}(\mathrm{wt} \%)=\left(\left(\mathrm{W}_{1}-\mathrm{W}_{0}\right) / \mathrm{W}_{1}\right) \times 100$

where $\mathrm{W}_{0}$ and $\mathrm{W}_{1}$ are the weight of the dry and hydrated sample, respectively.

In hydrated polymers, EWC encompasses different types of water as expressed by equation (2):

$$
\begin{aligned}
& \text { EWC }(\mathrm{wt} \%)=N F W(\mathrm{wt} \%)+I W(\mathrm{wt} \%)+F W(\mathrm{wt} \%) \\
& I W(\mathrm{wt} \%)=\Delta H_{\mathrm{cc}} / 334\left(\mathrm{Jg}^{-1}\right) \\
& F W(\mathrm{wt} \%)=\left(\Delta \mathrm{H}_{\mathrm{m}} / 334\left(\mathrm{Jg}^{-1}\right)\right)-I W
\end{aligned}
$$

$\Delta H_{\mathrm{cc}}$ represents enthalpy change during cold crystallization whereas $\Delta H_{\mathrm{m}}$ is enthalpy change during the melting of ice. 3-7 mg of samples was taken. Before measurements, the samples were immersed in ultrapure water for 7 days to attain full hydration. 


\section{Preparation and characterization of the polymer surfaces}

Film samples were produced and utilized for contact angle (CAs) and atomic force microscopy (AFM) measurements as well as for the human blood platelet adhesion test. For this reason, the polymer samples H-PMEA and PMEA were dissolved in toluene $\left(60^{\circ} \mathrm{C}\right.$ for 3 days $)$ or methanol, respectively, to give $0.2 \mathrm{wt} / \mathrm{vol} \%$ solutions. Round-shaped PET surfaces with the diameter of 14 mm were washed with methanol, and the filtered polymer solutions were spin-coated onto them. A Mikasa spin coater MS-A100 was used to coat the substrates in the following manner: Any particular polymer solution was coated twice onto a substrate at rates of $500 \mathrm{rpm}$ for $5 \mathrm{~s}, 2000$ rpm for $10 \mathrm{~s}$, slope for $5 \mathrm{~s}, 4000 \mathrm{rpm}$ for $5 \mathrm{~s}$, and slope for $4 \mathrm{~s}$. Afterwards, the substrates were dried at room temperature.

Water CAs on the airside of the polymer films were measured by the sessile drop method with an accuracy of $\pm 2^{\circ}$ at $25^{\circ} \mathrm{C}$. An air bubble of $2 \mu \mathrm{L}$ was injected to the hydrated surface immersed in water in the captive bubble method, which is a means of measuring the contact angle between a liquid and a solid by the drop shape analysis. ${ }^{31,32}$ The averages of three measurements carried out at different positions of a film are reported for both measurements.

AFM observations were carried out with a Bioscope Resolve (Bruker) Instrument with a cantilever SNL-10 (spring constant $k=0.35 \mathrm{~N} / \mathrm{m}$, resonance frequency $\mathrm{f}=65 \mathrm{kHz}$ in air, tip radius <12 nm; Bruker). AFM topographies were imaged in PBS in a Peak Force 
Tapping mode after the samples had been immersed in PBS for 60 min or longer. The arithmetical mean height is determined before and after immersion in PBS for 60 minutes.

\section{Determination of the Amount of Adsorbed Protein by $\mu B C A$ and ELISA 1-3,27,28,30,31}

Amounts of the platelet poor plasma (PPP) adsorbed on the polymer surfaces were quantified by the $\mu \mathrm{BCA}$. In addition, the ELISA was executed to relatively evaluate the conformational alteration of the adsorbed fibrinogen from PPP.

The polymer surfaces for the $\mu \mathrm{BCA}$ and the ELISA were prepared as follows: a 96-well polypropylene plate was coated with the polymers by applying $15 \mu \mathrm{L}$ of the polymer solution $(0.2 \mathrm{wt} / \mathrm{vol} \%)$ of PMEA in methanol or of H-PMEA in toluene, and slowly air-drying the surface at room temperature for 3 days.

For both methods, $100 \mu \mathrm{L}$ of PBS was introduced in each well followed by incubating the plate for 1 hour at $37^{\circ} \mathrm{C}$. The PBS was removed, $50 \mu \mathrm{L}$ of PPP was put on in each well, and the plate was incubated again for another hour at the same temperature. PPP was removed from each well, and the wells were rinsed with $200 \mu \mathrm{L}$ PBS for at least five times. In case of the $\mu \mathrm{BCA}$, a $30 \mu \mathrm{L}$ solution of $5 \%$ sodium dodecyl sulfate and $0.1 \mathrm{~N} \mathrm{NaOH}$ was used to extract the adsorbed proteins by incubation for 2 hours at $37^{\circ} \mathrm{C}$. The proteins were then evaluated by the $\mu \mathrm{BCA}$ assay 
following the manufacturer's instructions. The protein amount was determined by making use of the albumin standard curve that had been prepared for each experiment.

When the ELISA was applied the procedure, in the beginning was the same as above using PPP. The samples were then incubated with Blocking-One (Nacalai Tesque) for $30 \mathrm{~min}$ at the same temperature preventing non-specific reactions. Washing five times with $200 \mu \mathrm{L}$ PBS as before followed. Further incubation with $50 \mu \mathrm{L}$ of Anti-Fibrinogen $\gamma^{\prime}$ antibody (Ab) (Millipore) for 1 hour at $37^{\circ} \mathrm{C}$, and then (after washing) with peroxidaseconjugated anti-mouse Immunoglobulin $\mathrm{G}(\operatorname{lgG})$ Ab for 2 hours at $37^{\circ} \mathrm{C}$ followed. Lastly, the samples were incubated with 2,2'-azinobis(3-ethylbenzothiazoline-6-sulfonic acid)ammonium salt in a buffer (Roche Diagnostics). A plate reader (BIO-RAD) was used to measure absorbance at a wavelength of $405 \mathrm{~nm}$. The averages of 5 measurements are reported.

\section{Human Platelet Adhesion Test'-3,27,28,30,31}

The polymer substrates for the platelet adhesion test with human blood were fabricated in the following manner: $8 \times 8 \mathrm{~mm}$ squares were cut out of the polymer 
coated substrates, and were mounted on an SEM specimen stage with a double-sided tape. Platelet-rich plasma (PRP) and PPP were separated from human blood by centrifuging in two stages: the first stage involved centrifuging at $1500 \mathrm{rpm}$ for $5 \mathrm{~min}$ to get PRP while the second stage involved centrifuging the same blood at $4000 \mathrm{rpm}$ for 10 min to get PPP. The cells in PRP and PPP were counted on a hemocytometer, and a plasma solution comprising $4 \times 10^{7}$ cells $\mathrm{cm}^{-2}$ of platelets was obtained by mixing the PRP and PPP solutions in a predetermined ratio. $200 \mu \mathrm{L}$ of the final solution was applied onto the polymer-coated substrates, which afterwards were washed with PBS twice. Immersion in $1 \%$ glutaraldehyde in PBS for 120 min at $37^{\circ} \mathrm{C}$ ensured fixation of the adhered platelets. As the final step, the substrates were washed in this order: once with PBS, once with PBS-water 1:1 mixture, and twice with pure water. The substrates were examined with SEM to determine the number of adhered platelets. Human blood obtained from 3 different donors was used, and the platelet adhesion test was carried out 3 times for statistical purpose. The averages of 5 points measured on 3 different films are reported. 


\section{Results and discussion}

A copolymer of MEA with the functional methacrylic monomer 2-\{3-(6-methyl-4-oxo-1,4dihydropyrimidin-2-yl)ureido $\}$ ethyl methacrylate ${ }^{8,9,33}$ was synthesized as reported by Shabir et. al. ${ }^{29}$ The copolymer contained about $6 \mathrm{~mol} \%$ of the functional monomer as judged by ${ }^{1} \mathrm{H}$ NMR (Figure S1). The structure of the copolymer is depicted also in Scheme 1a. Because of the multiple hydrogen bonding, the copolymer was designated as hydrogen-bonding PMEA (HPMEA). This kind of bonding between the copolymer chains is realized through stronglyassociating stickers (Scheme 1c and 1d). Introducing such stickers turns liquid PMEA into solid, rubber-like, and not sticky H-PMEA, which can be processed into a transparent self-supporting film shown in Scheme 1b. Films of various thicknesses can be produced by pressing the material at $60{ }^{\circ} \mathrm{C}$ and $5 \mathrm{MPa}$, or by solvent casting.

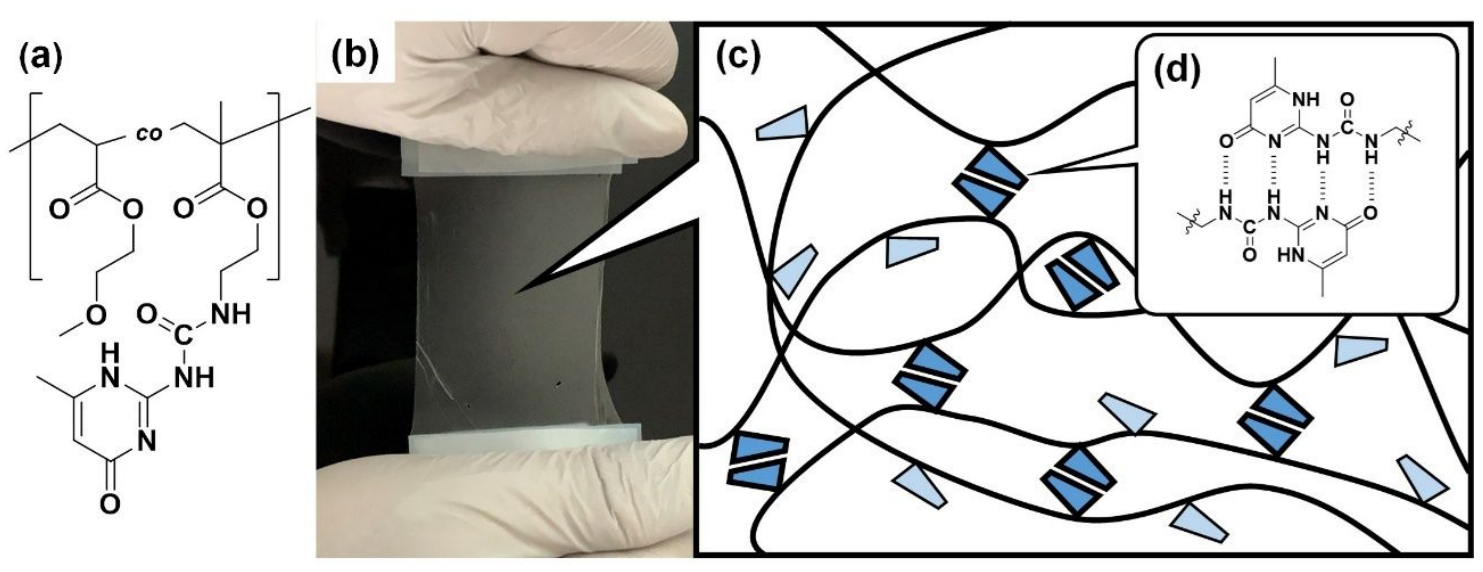

Scheme 1. Chemical structure of poly[2-methoxyethyl acrylate-co-2-\{3-(6-methyl-4-oxo1,4-dihydropyrimidin-2-yl)ureido\}ethyl methacrylate] (H-PMEA) (a), appearance of the 
polymer as a transparent self-supporting film (b), morphology with stickers (c), and multiple hydrogen bonding at the side chains (d).

\section{Thermal and mechanical properties}

Besides being solid, H-PMEA features thermal stability. The overlay of the TGA curves of PMEA and H-PMEA in air and nitrogen is shown in Figure S1. The initial degradation of HPMEA starts at around $30{ }^{\circ} \mathrm{C}$ later than that of PMEA, and it occurs in 2 steps. Having in mind the high amount of water that is associated with the MEA polymers even in dry conditions it is assumed that the process of dehydration for both polymers finishes at $300^{\circ} \mathrm{C}$. The second step is related to the main degradation, where H-PMEA decomposes at a higher temperature than PMEA. Thus, H-PMEA is more thermally stable than PMEA, a property gained by forming physical cross-links.

For comparison, PVC degrades in 3 steps and at $300{ }^{\circ} \mathrm{C}$ already $45 \%$ to $50 \%$ of the polymer is thermally decomposed.

The rheological properties of the copolymers of MEA and the functional methacrylic monomer bearing a 6-methyl-2-ureido-4[1 $\mathrm{H}]$-pyrimidone group (3 or $8 \mathrm{~mol} \%$ of the functional monomer) have already been evaluated. ${ }^{29}$ We investigate the basic mechanical properties of the H-PMEA (with $6 \mathrm{~mol} \%$ of the functional monomer) synthesized for this work and PVC. Tensile stress measurements and DMA were 
performed at room temperature. The film thickness was 0.1 and $0.2 \mathrm{~mm}$ for H-PMEA and PVC, respectively. Big differences in the elastic modulus (0.3 and $220 \mathrm{PMa}$ ), tensile stress (1.1 and 57.2 MPa), and elongation (814 and 8\%) of H-PMEA and PVC films were observed. The elastic modulus and the $T_{g}$ of H-PMEA, determined by DMA are close to those from the tensile stress and DSC measurements (Table 1). These results show that although being of lower strength, H-PMEA is a rubber-like polymer, which can be employed to form thick coatings. Furthermore, the acquired mechanical data confirm, that this polymer is able to withstand the necessary loads for forming free-standing films and articles, possibly with various forms and dimensions. The MW and the number of hydrogen-bonding stickers in the H-PMEA can be adjusted further to obtain particular target properties of the material.

\section{Hydration structure of PMEA and H-PMEA}

The hydration structure of H-PMEA is evaluated by DSC, AFM, and contact angle (CA) measurements, and compared to PMEA with a similar MW (Table 1). The $T_{\mathrm{g}}$ of both polymers in a dry state are quite close, however, they differ a lot in the hydrated 
state. That PMEA exhibits $15{ }^{\circ} \mathrm{C}$ lower $T_{\mathrm{g}}$ in its hydrated state is already known. It is related to the polymer-water interactions that will be elaborated later in this paragraph. $T_{\mathrm{g}}$ of H-PMEA in a hydrated state has decreased by only $8^{\circ} \mathrm{C}-$ the hydrogen bonding and thereby lower mobility restrict the diffusion of water, and therefore its interaction with the high viscosity polymer is weakened. However, the $T_{\mathrm{g}}$ of the copolymer is still below room temperature, $-33.5^{\circ} \mathrm{C}$.

The CA measurements show that the intermediately hydrophilic PMEA is transformed into an intermediately hydrophobic H-PMEA polymer (Table 1). While the water CA (by sessile drop method) is stable in case of PMEA $\left(42^{\circ}\right)$, it continues changing with time and from $65^{\circ}$ at 30 min reaches $58^{\circ}$ at 60 min for H-PMEA. The air CA (by captive bubble method) is stable for both polymers and decreases from 142 to only $140^{\circ}$ for $\mathrm{H}$ PMEA during the 60 min scan - this polymer needs longer time to form its interfacial structure with water. However, after immersing 24 hours in water there is no more change in the air CA of H-PMEA. All these show that the copolymerization and the physical cross-links have turned PMEA into more hydrophobic H-PMEA with lower mobility - a feature responsible for the platelet adhesion onto a polymer. 
Based on its interactions with a polymer, 3 kinds of water in macromolecules are classified: non-freezing water (NFW), intermediate water (IW), and free water $(F W)$. In this order the polymer-water interactions decrease, and therefore also the freezing bound water is referred to as $I W$ as it has an intermediate association with the polymer matrix. It is believed, that polymer's blood compatibility is related to the amount of $I W$. The water content of both polymers is therefore investigated by DSC, and the results are summarized in Table 2. Additionally, the DSC curves are shown in Figure S2. In the cooling scan, only the $T_{g}$ of H-PMEA could be detected. In contrast, PMEA shows $I W$ already in the cooling scan, whereas in case of H-PMEA it is observed in the heating scan and with much lower content. This behaviour is characteristic of polymers with lower mobility. Moreover, EWC of H-PMEA is quite different from that of PMEA; in the former, this amount is more than twice decreased as the physical cross-links lead to a reduction in free volume and a reduction in the polymer chain mobility. This prevents water from easily entering into the free volume of the polymer. More data presented in Table 2 show that this has happened by decreasing primarily $F W$ (by $90 \%$ ) and $I W$ (by 40\%) in H-PMEA. The $\mathrm{H}$-bonding present in the polymer matrix increases the 
interactions between macromolecules and decreases those between water and the polymer chains: IW and NFW predominate in PMEA as compared to H-PMEA. This influences FWas well, which is being 10 times less in H-PMEA than in PMEA. The IWC is also lower in H-PMEA as compared to the NFWC, possibly due to the added interactions of its cross-links with water resulting in more NFW. These results are expected to influence the platelet adhesion on the surface of H-PMEA too.

Table 2. Water content of the polymers

\begin{tabular}{|l|c|c|c|c|c|}
\hline Polymer & EWC & NFWC & IWC & FWC & IWC/NFWC \\
wt $\%$ & wt $\%$ & wt\% & wt $\%$ & \\
\hline PMEA & 9.6 & 1.8 & 3.8 & 4.0 & 2.1 \\
\hline H-PMEA & 4.3 & 1.6 & 2.3 & 0.4 & 1.4 \\
\hline
\end{tabular}

\section{AFM of the hydrated polymers}

The interface morphology of the samples in dry and hydrated states was evaluated by AFM. The AFM images of the dry polymers (not shown here), PMEA ${ }^{34-36}$ and H-PMEA, 
present flat structures with an arithmetical mean height around $5 \mathrm{~nm}$. By immersing the substrate with the polymers in PBS, continuous hydration of the surface induces phase separation forming a nanoscale structure. Therefore, in the AFM images of the hydrated polymers protrusions appear due to the micro-phase separation into polymer-rich and water-rich domains. ${ }^{34-36}$ Such structures of polymer and water at the interfacial region are characteristic for biopolymers containing a large amount of $I W$. These interfacial nanometer-scale structures in case of both investigated polymers have similar shapes and are with similar domain sizes (Figure 1). The height of the protrusions for H-PMEA has the same range as previously found for PMEA: 50 - 80 microns. ${ }^{1-3,6,34-36}$ However, the numbers of the soft protrusions/hills seem to be more and at the same time the areas of the valleys are smaller in H-PMEA as compared to PMEA, probably because of these two being hydrophobic and hydrophilic, respectively. These facts resulted in the less pronounced phase separation of H-PMEA clearly visible in its $2 \mathrm{D}$ and $3 \mathrm{D}$ images. The H-bonding obstructs segregation of the MEA polymer at the polymer-PBS interface, which results in the possibility for the blood cells to settle down probably on the hills. ${ }^{34-36}$ 
HPMEA
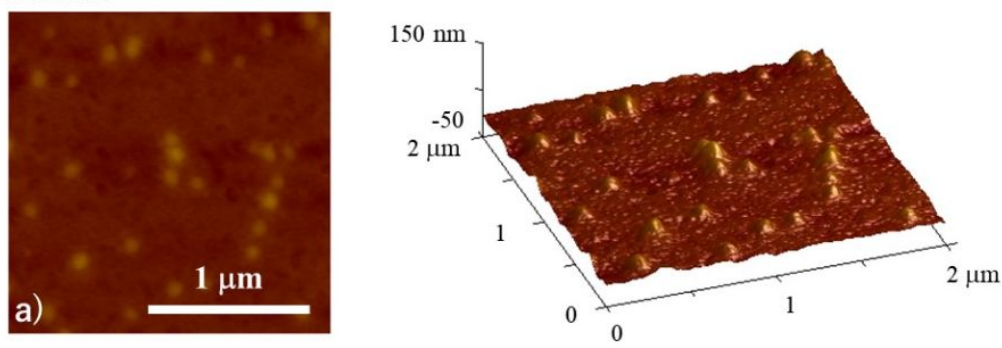

$\operatorname{PMEA}\left(M_{\mathrm{n}}=19 \mathrm{~kg} / \mathrm{mol}\right)$
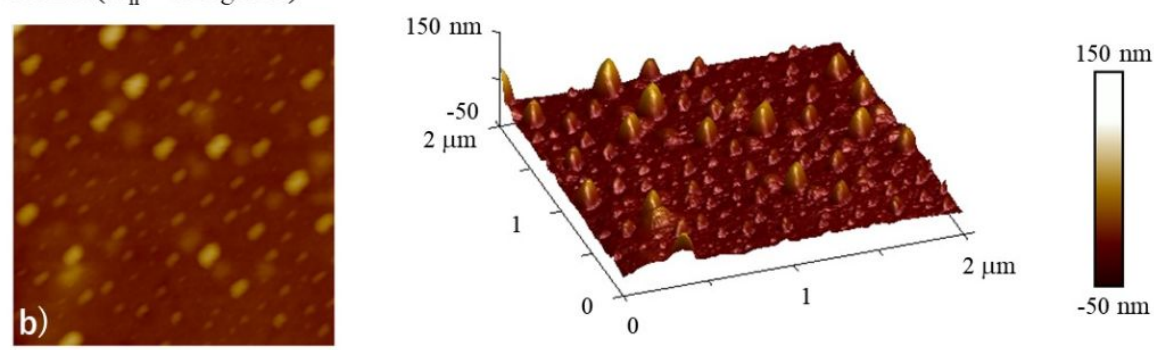

Figure 1. Two- (left) and three-dimensional (right) images of the polymer/PBS interfaces

of H-PMEA (a) and PMEA (b)

Protein adsorption and denaturation behavior on MEA polymers and negative control

substrates

Plasma proteins including fibrinogen and albumin adsorb easily to the material surface

through nonspecific interactions, and the cell adhesion behavior depends strongly on the amount, the composition, and the structure of adsorbed proteins. Thus, its surface adsorption amount was analyzed by a micro BCA using PPP. The result is shown in 
Figure 2. A significant change was observed for the adsorbed proteins from PPP onto PMEA and H-PMEA, probably due to the effect of physical crosslinking that restrict the mobility of polymer chains and reduces the amount of IWC. Even though H-PMEA adsorbs more proteins than PMEA, their amount is much less than the ones adsorbed onto PP and especially PVC.

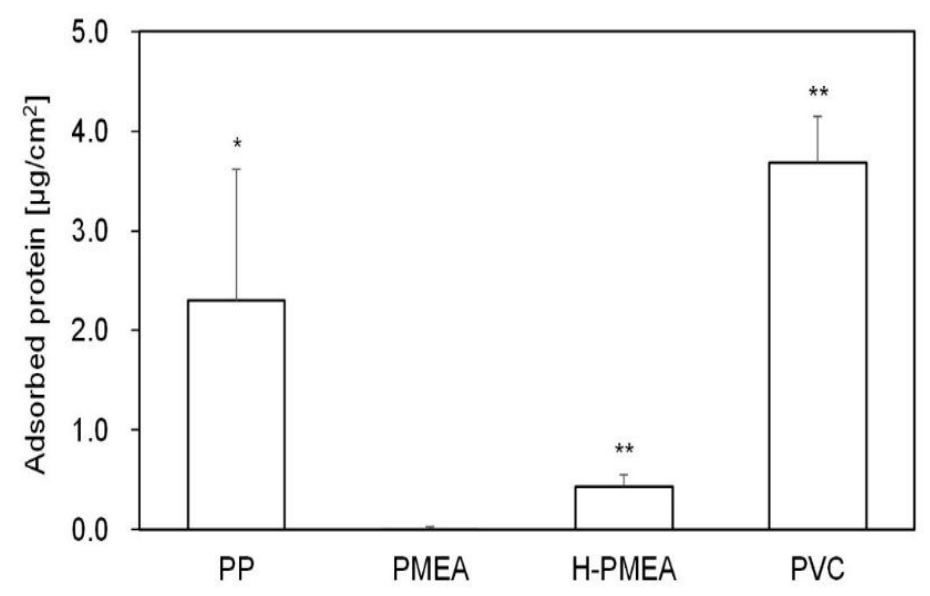

Figure 2. Amount of adsorbed proteins determined by the micro BCA method with PPP. The data represent the mean $\pm S D(n=5) . \star \star: P<0.01$ vs. PMEA, and $\star: P<0.05$ vs. PMEA. PP and PVC are used as controls.

Subsequently, the conformational alteration of adsorbed fibrinogen was quantified by an ELISA. The plasma proteins change in conformation due to the contact with the 
foreign body surface. The conformational alteration exposes the platelet adhesion site in fibrinogen, and the exposure degree of this site on the material surface has a direct impact on the non-thrombogenic property of materials. Thus, the ELISA with the antibody that can identify c-terminus in the fibrinogen $y^{\prime}$ chains - the platelet adhesion site - was employed to evaluate the degree of the site exposure using PPP. The results are presented in Figure 3. There was no significant difference between PMEA and $\mathrm{H}-$ PMEA, and the conformation of adsorbed fibrinogen remained unchanged against the adsorption onto both MEA polymer surfaces. In contrary, this is not the case for the negative controls, PP and PVC, where even for PVC the conformational alteration of fibrinogen is almost doubled as compared to PP (Figure 4).

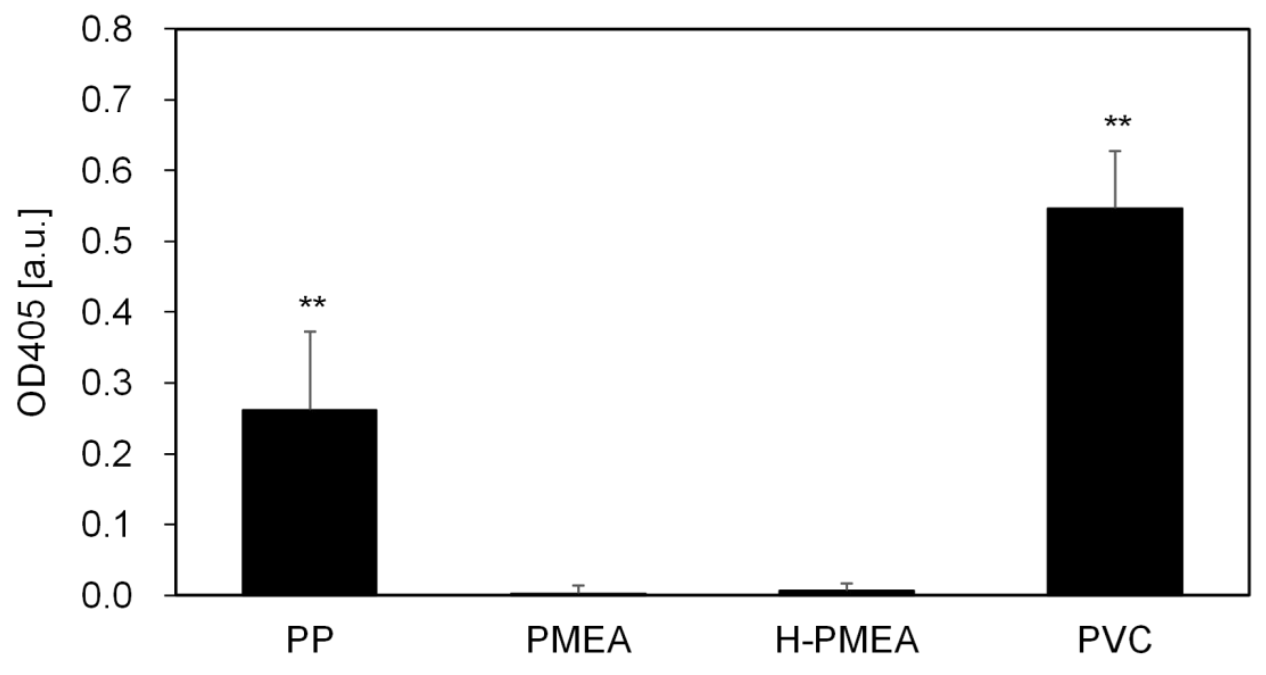


Figure 3. Conformationally altered fibrinogen in the adsorbed proteins evaluated by the ELISA method with PPP detected with an anti-fibrinogen $\gamma^{\prime}$ chain antibody after $1 \mathrm{~h}$. The data represent the mean \pm SD $(n=5) . \star \star: P<0.01$ vs. PMEA. PP and PVC are used as controls.

\footnotetext{
Analyses with human blood

Platelet adhesion test with human blood is the best direct method to reveal the blood compatibility of polymers. As depicted in Figure 4, the blood compatibility of H-PMEA is slightly decreased as compared to that of PMEA, probably due to its higher hydrophobicity, lower water and $I W$ content, and chain mobility. Also, the free volume of the polymers is expected to play a role, as the H-bonding should reduce the distances between 2 sliding macromolecules. However, the decrease of the platelet adhesion on both MEA polymers as compared to PET and PVC is enormous. These results further substantiate the anticipated big advantage of the examined H-PMEA over the other hydrophobic polymers used as negative control (Figure 2-4).
} 


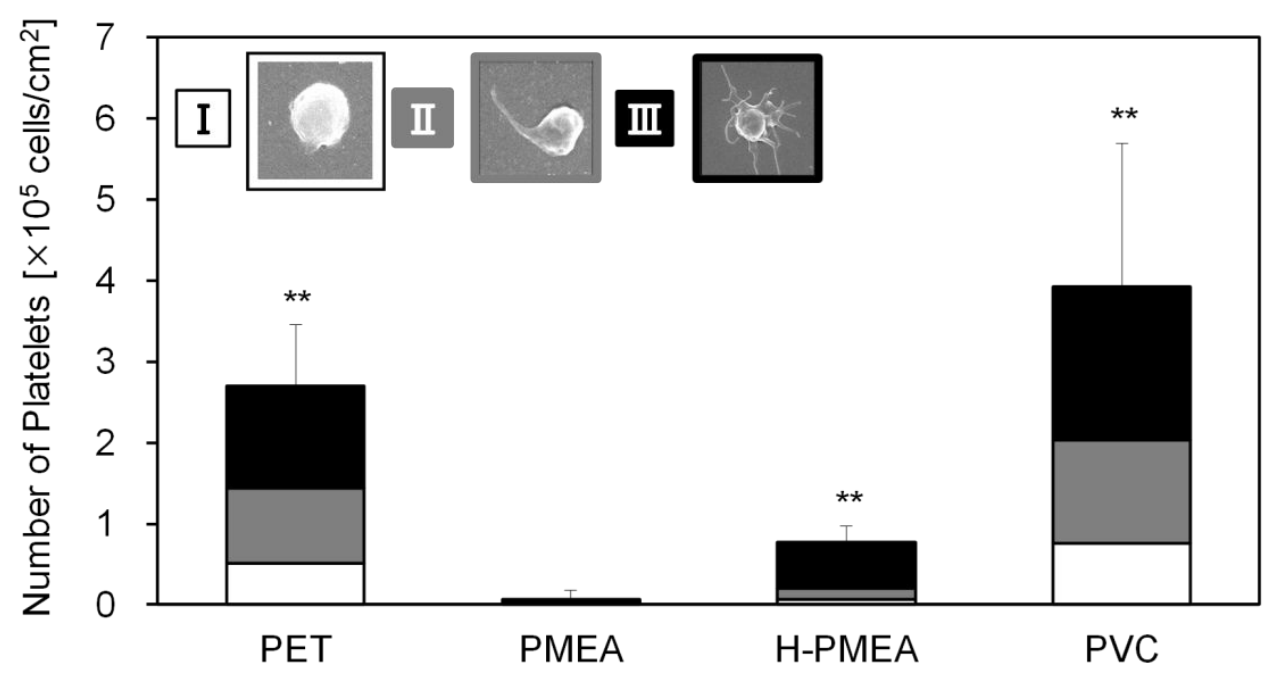

Figure 4. Platelet adhesion test with human blood onto PMEA and H-PMEA as compared to PET and PVC. Blood of three donors was tested in triplicates, and five fields were analyzed per substrate. The data represent the mean $\pm S D$. $\star \star: ~ P<0.01$ vs. PMEA. PP and PVC were used as controls. The number of adhered platelets was classified into three types according to the degree of activation as shown in the figure: (I) native (spherical shape), (II) partially activated (hemispherical shape with several pseudopods), and (III) activated (spread shape with numerous pseudopods or flat discoid).

The morphology of the platelets (Figure 4) was observed by SEM (Figure 5). The platelet adhesion was suppressed by both PMEA and H-PMEA films onto PET, 
compared with that of untreated PET and PVC. The adhered platelets on all surfaces except PMEA formed many pseudopods and spread, which means that even though there was a low number onto H-PMEA film, more than half of the platelets were highly activated. PET and PVC have much higher numbers of adhered platelets, 33 and $48 \%$ of which show the highest activation degree III.
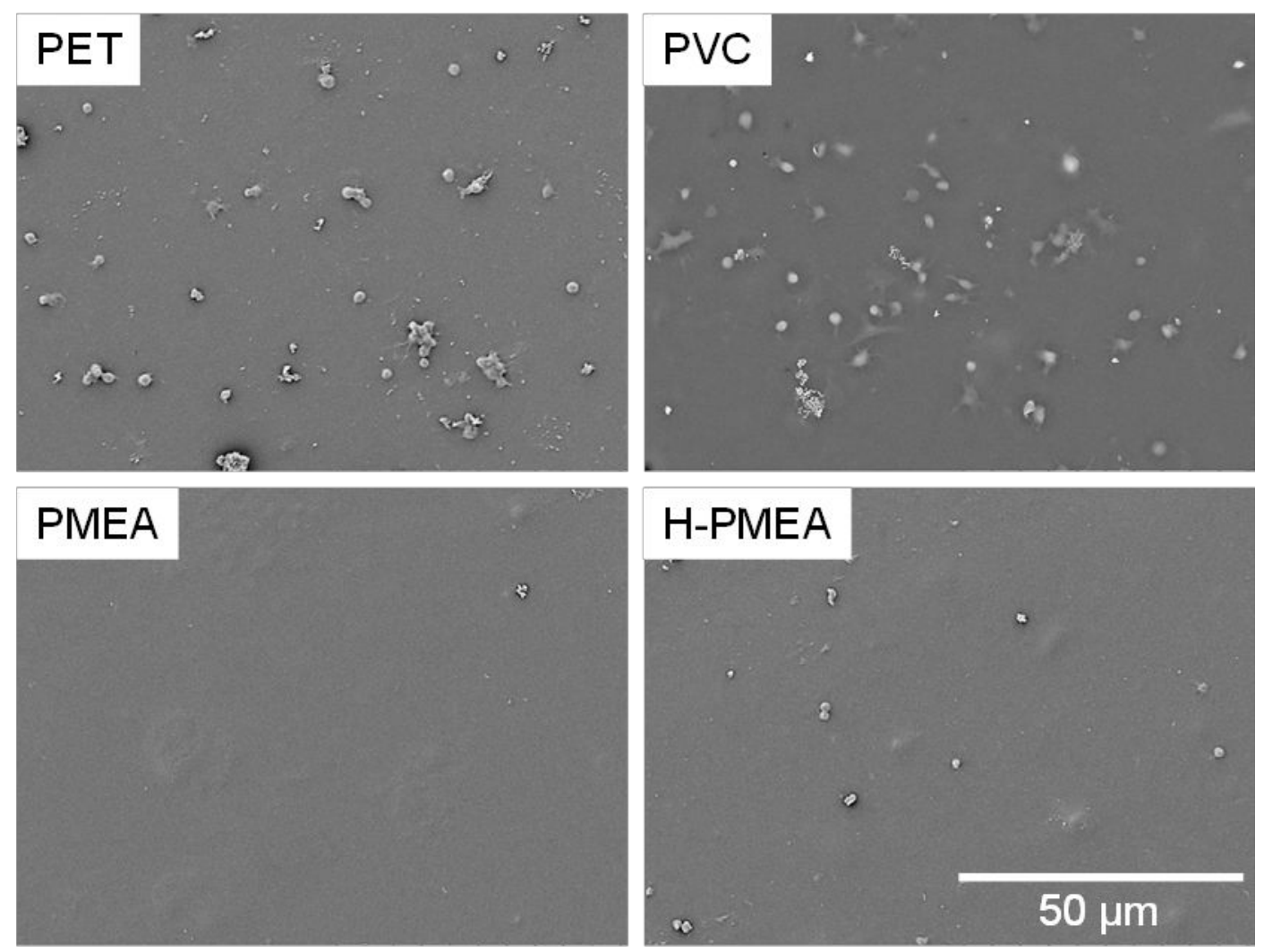
Figure 5. Scanning electron micrographs of platelets adhered onto the surface of PET, PMEA, H-PMEA, and PVC. The scale bare corresponds to $50 \mu \mathrm{m}$ in all micrographs.

The trend for the platelet compatibility of the MEA polymers evaluated in this work is the same in all methods employed: ELISA, micro BCA, and human blood test. Normalized values of the protein adsorption of the MEA polymers to that of PVC, presented in Figure 6, suggest that the most blood compatible is PMEA, followed by $\mathrm{H}$ PMEA. In this order, the water content of the polymers and their IWC, as well as the mobility and the hydrophilicity decreases, which explains the slightly decreased blood compatibility of H-PMEA. This observation is in accord with the morphology/topography of hydrated PMEA and H-PMEA obtained by AFM. Presence of more polymer-rich protrusions and less water-rich valleys in hydrated H-PMEA results in H-PMEA being slightly less blood compatible than PMEA as the protrusions are believed to serve as scaffolds for fibrinogen (a protein playing an important role in thrombus formation) adsorption and the valleys are expected to comprise much of $I W^{34-36}$ Therefore, these 
parameters can be used as a quantitative guidepost for the blood compatibility of various materials.

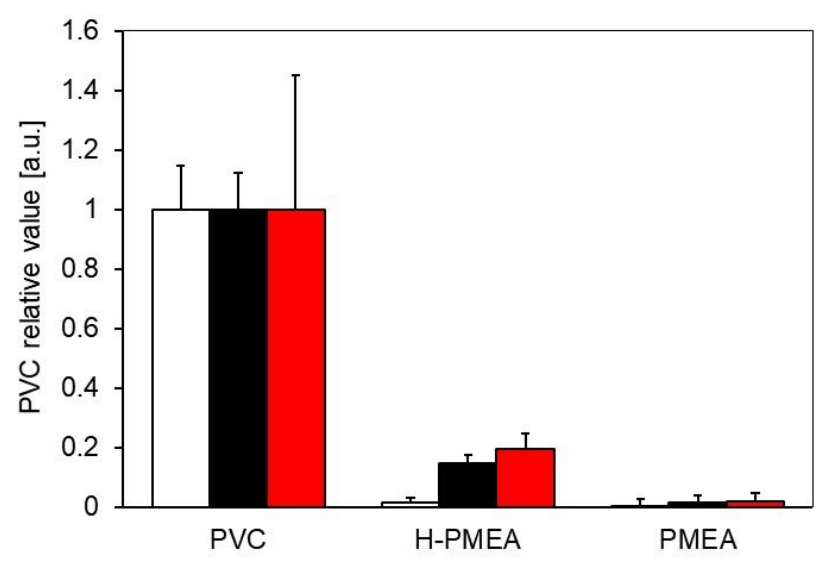

Figure 6. Normalized values of the conformational alteration of adsorbed fibrinogen evaluated by ELISA (white), the amount of adsorbed protein from PPP evaluated by $\mu-$ $\mathrm{BCA}$ (black), and the number of adhered platelets by human platelet adhesion test (red) to those of PVC.

Despite slight decrease in blood compatibility, the gain from turning the viscous PMEA to solid H-PMEA is enormous: preparation of free-standing films, thick coatings, possibly articles with various shapes and geometries for the medicinal and anti-fouling industry with still significantly higher compatibility with human blood as compared to PVC. 


\section{Conclusion}

In conclusion, introducing strongly-associating hydrogen-bonding stickers in PMEA

resulted in transforming this low viscosity polymer featuring excellent blood compatibility

into a solid, rubber-like, and thermally stable supramolecular copolymer H-PMEA. Blood

compatibility is assessed by 3 methods showing either no or slightly increased

adsorption of platelets onto the rubbery H-PMEA when comparing it with the low

viscosity PMEA. These changes in case of H-PMEA may be attributed to its higher

hydrophobicity, lower chain mobility in hydrated condition, lower water, $F W$, and $I W$

content as compared to the MEA homopolymer. Analysis of the free volume of both

MEA polymers, which should also influence the water-polymer interactions at the

interface, is being carried out. While sacrificing a bit the blood/platelet compatibility of

PMEA, H-PMEA allows for the preparation of free-standing films and thick coatings as

well as potentially tubes and articles with various shapes and geometries for the

medicinal and anti-fouling industry with still significantly higher compatibility with human

blood as compared to PVC. Thus, H-PMEA displays promising properties as the

material that may replace polymers in products used in contact with blood or storage of 
blood, which either are expensive or are known to comprise harmful for our health compounds. The period of implementation during storage and processing can be at the same time extended, the platelet adhesion dramatically suppressed, and the material recycled. This strategy will be implemented in our laboratories to turn other viscous blood compatible polymers into solid materials as well.

\section{ASSOCIATED CONTENT}

\section{Supporting Information}

Supporting Information. ${ }^{1} \mathrm{H}$ NMR spectroscopy, TGA, and DSC results for the discussed polymers.

The Supporting Information is available free of charge on the ACS Publication website at DOI: ${ }^{* * *}$.

\section{AUTHOR INFORMATION}

Corresponding Author 
*E-mail: masaru_tanaka@ms.ifoc.kyushu-u.ac.jp

ORCID

Katja Jankova: 0000-0002-4218-064X

Irakli Javakhishvili: 0000-0003-3681-3161

Shingo Kobayashi: 0000-0002-8357-8654

Ryohei Koguchi: 0000-0003-0017-4783

Daiki Murakami: 0000-0002-5552-4384

Toshiki Sonoda: 0000-0002-0504-7559

Masaru Tanaka: 0000-0002-1115-2080

\section{Notes}

The authors declare no competing financial interest. 


\section{ACKNOWLEDGEMENTS}

Project PROGRESS 100 from Kyushu University is acknowledged for financial

support. This work was partially supported by Dynamic Alliance for Open Innovation Bridging Human, Environment and Materials.

\section{REFERENCES}

(1) Tanaka, M.; Motomura, T.; Kawada, M.; Anzai, T.; Kasori, Y.; Shiroya, T.; Shimura, K.; Onishi, M.; Mochizuki, A. Blood compatible aspects of poly(2-methoxyethylacrylate) (PMEA) - relationship between protein adsorption and platelet adhesion on PMEA surface. Biomaterials 2000, 21 (14), 1471-1481.

(2) Tanaka, M.; Motomura, T.; Kawada, M.; Anzai, T.; Kasori, Y.; Shimura, K.; Onishi, M.; Mochizuki, A.; Okahaya, Y. A new blood-compatible surface prepared by poly(2methoxyethylacrylate)(PMEA) coating-protein adsorption on PMEA surface. Jpn. J. Artif. Organs 2000, 29, 209-216.

(3) Tanaka, M.; Mochizuki, A.; Ishii, N.; Motomura, T.; Hatakeyama, T. Study of Blood Compatibility with Poly(2methoxyethyl acrylate). Relationship between Water Structure and Platelet Compatibility in Poly(2-methoxyethyl acrylate-co-2hydroxyethyl methacrylate). Biomacromolecules 2002, 3(1), 36-41. 
(4) Anzai, T.; Okumura, A.; Kawaura, M.; Yokoyama, K.; Oshiyama, H.; Kido, T.; Nojiri, C. Evaluation of the Biocompatibility of an In Vitro Test Using a Poly(2methoxyethylacrylate) Coated Oxygenator. Jpn. J. Artif. Organs 2000, 9, 73-77.

(5) Lee, W.; Kobayashi, S.; Nagase, M.; Jimbo, Y.; Saito, I.; Inoe, Y.; Yambe, T.; Sekino, M.; Malliaras, G.G.; Yokota, T.; Tanaka, M.; Someya, T. Nontrombogenic, stretchable, active multielectrode array for electroanatomical mapping. Science Advances 2018, 4:eaau2426.

(6) Murakami, D.; Mawatari, N; Sonoda, T.; Kawazaki, A.; Tanaka, M. Effect of the molecular weight of Poly(2-methoxyethyl acrylate) on Interfacial Structure and Blood Compatibility. Langmuir 2019, 35, 2808-2813.

(7) Kureha, T.; Hiroshige, S.; Matsui, S.; Suzuki, D. Water-immiscible bioinert coatings and film formation aqueous dispersions of poly(2-methoxyethyl acrylate) microspheres. Colloids Surf. B 2017, 155, 166-172.

(8) Bottehuis, N.E.; van Beek, D.J.M.; van Gemert, G.M.L.; Bosman, A.W.; Sijbesma, R.P. Self-assembly and morphology of polydimethylsiloxane supramolecular thermoplastic elastomers, J. Polym. Sci., Part A: Polym. Chem. 2008, 46 (12), 3877-3885.

(9) Yamauchi, K.; Lizotte, J.R.; Long, T.E. Thermoreversible poly(alkyl acrylates) consisting of self-complementary multiple hydrogen bonding. Macromolecules 2003, 36 (4), 10831088.

(10) Ogliani, E.; Yu, L.; Javakhishvili, I.; Skov, A.L. A thermo-reversible silicone elastomer with remotely controlled self-healing. $R S C A d v$. 2018, 8, 8285-8291.

(11) Chen, S.; Mahmood, N.; Beiner, M.; Binder, W.H. Self-Healing Materials from V- and H-Shaped Supramolecular Architectures. Angew. Chem. Int. Ed. 2015, 54, 10188-10192 
(12) Kajita, T.; Noro, A.; Mtsushita, Y. Design and properties of supramolecular elastomers. Polymer 2017, 128, 297-310.

(13) Weber, M.J.; Dankers, Y.W. Supramolecular hydrogels for biomedical applications. Macromol. Biosci. 2019, 19, 1800452.

(14) Pan, Y.; Hu, J.; Yang, Z.; Tan, L. From Fragile Plastic to Room-Temperature SelfHealing Elastomer: Tuning Quadruple Hydrogen Bonding Interaction through One-Pot Synthesis. ACS Appl. Polym. Mater. 2019, DOI:10.1021/acsapm.8b00153

(15) Dimitrov, I.; Jankova, K.; Hvilsted, S. Synthesis of Polystyrene-Based Random Copolymers with Balanced Number of Basic or Acidic Functional Groups. J. Polym. Sci., Part A: Polym. Chem. 2010, 48, 2044-2052.

(16) Kerres, J.; Ullrich, A.; Haring, T.; Baldauf, M.; Gebhardt, U.; Preidel, W. Preparation, characterization and fuel cell application of new acid-base blend membranes. J. New Mater. Electrochem. Syst. 2000, 3 (3), 2000, 229-239.

(17) Li, W.; Manthiram, A.; Guiver, M.D. Acid-base blend membranes consisting of sulfonated poly(ether ether ketone) and 5-amino-benzotriazole tethered polysulfone for DMFC. J. Membrane Sci. 2010, 362, 289-297.

(18) Hietala, S.; Mononen, P.; Strandman, S.; Järvi, P.; Torkkeli, M.; Jankova, K.; Hvilsted, S.; Tenhu, H. Synthesis and rheological properties of an associative star polymer in aqueous solutions. Polymer 2007, 48, 4087-4096.

(19) Hietala, S.; Strandman, S.; Järvi, P.; Torkkeli, M.; Jankova, K.; Hvilsted, S.; Tenhu, H. Rheological Properties of Associative Star Polymers in Aqueous Solutions: Effect of Hydrophobe Length and Polymer Topology. Macromolecules 2009, 42 (5), 1726-1732. 
(20) Jankova, K.; Jannasch, P.; Hvilsted, S. Ion conducting solid polymer electrolytes based on polypentafluorostyrene- $b$-polyether- $b$-polypentafluorostyrene prepared by atom transfer radical polymerization J. Mater. Chem. 2004, 14, 2902-2908.

(21) Jannasch, P. Ionic conductivity in physical networks of polyethylene-polyetherpolyethylene triblock copolymers Chem. Mater. 2002, 14, 2718-2724.

(22) Haishima, Y.; Kawakami, T.; Fukui, C.; Tanoue, A. Characterization of alternative plasticizers in poly(vinyl chloride) sheets for blood containers. J. Vinyl Addit. Techn. 2015, $22(4), 520-528$.

(23) www.renolit.com: Blood and blood component applications.

(24) www.melitek.com; www.pvcfreebloodbag.eu: New PVC-free blood bags have reached a major milestone, 2015.

(25) Bhaskaran Nair, C.S.; Vidya, R.; Ashalatha, P.M. Hexamoll DINCH plasticised PVC containers for the storage of platelets. Asian J. Transfus. Sci. 2011, 5, 18-22.

(26) Dumont, L.J.; VandenBroeke, T. Seven-day storage of apheresis platelets: report on an in vitro study. Transfusion 2003, 43, 143-150.

(27) Tanaka, M.; Mochizuki, A. Effect of water structure on blood compatibility - thermal analysis of water in poly(meth)acrylate. J. Biomed. Mater. Res. 2004, 68A (4), 684-695.

(28) Sato, K.; Kobayashi, S.; Sekishita, A.; Wakui, M.; Tanaka, M. Biomacromolecules 2017, 18, 1609-1616.

(29) Shabbir, A.; Javakhishvili, I.; Cerveny, S.; Hvilsted, S.; Skov, A.L.; Hassager, O.; Alvarez, N. Linear Viscoelastic and Dielectric Relaxation Response of Unentangled UPyBased Supramolecular Networks. Macromolecules 2016, 49, 3899-3910. 
(30) Koguchi, R.; Jankova, K.; Tanabe, N.; Amino, Y.; Hayasaka, Y; Kobayashi, D.; Miyajima, T.; Yamamoto, K.; Tanaka, M. Controlling the hydration structure with small amount of fluorine to produce blood compatible fluorinated poly(2-methoxyethyl acrylate). Biomacromolecules, 2019, 20, 2265-2275.

(31) Kobayashi, S.; Wakui, M.; Iwata, Y.; Tanaka, M. Poly( $\omega$-methoxyalkyl acrylate)s: Nonthrombogenic Polymer Family with Tunable Protein Adsorption. Biomacromolecules 2017, $18(12), 4214-4223$.

(32) Sato, K.; Kobayashi, S.; Sekishita, A.; Wakui, M.; Tanaka, M. Synthesis and Thrombogenicity Evaluation of Poly(3-methoxypropionic acid vinyl ester): A Candidate for Blood-Compatible Polymers. Biomacromolecules 2017, 18 (5), 1609-1616.

(33) Kajita, T.; Noro, A.; Matsushita, Y. Design and properties of supramolecular elastomers. Polymer 2017, 128, 297-310.

(34) Murakami, D.; Kobayashi, S.; Tanaka, M. Interfacial Structures and Fibrinogen Adsorption at Blood- Compatible Polymer/Water Interfaces. ACS Biomater. Sci. Eng. 2016, 2 (12), 2122-2126.

(35) Murakami, D.; Kitahara, Y.; Kobayashi, S.; Tanaka, M. Thermosensitive Polymer Biocompatibility based on Interfacial Structure at Biointerface, ACS Biomater. Sci. Eng. 2018, 4, 1591-1597.

(36) Ueda, T.; Murakami, D.; Tanaka, M. Analysis of interaction between interfacial structure and fibrinogen at blood-compatible polymer/water interface. Front. Chem. 2018, 6, 542: doi: $10.3389 /$ fchem.2018.00542. 


\section{Table of content graphics}

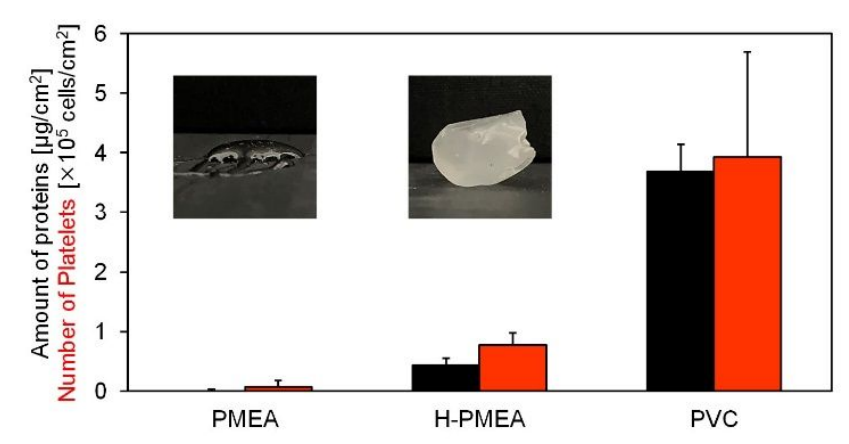

